\title{
Maximum Norm Stability of Difference Approximations to the Mixed Initial Boundary-Value Problem for the Heat Equation*
}

\author{
By J. M. Varah
}

\begin{abstract}
We consider the heat equation $u_{t}=u_{x x}$ in the quarter-plane $x \geqq 0, t \geqq 0$ with initial condition $u(x, 0)=f(x)$ and boundary condition $\alpha u(0, t)+u_{x}(0, t)=0$. We are concerned with the stability of difference approximations $v_{\nu}{ }^{n+1}=Q v_{\nu}{ }^{n}$ to this problem. Using the resolvent operator $(Q-z I)^{-1}$, we give sufficient conditions for consistent, onestep explicit schemes to be stable in the maximum norm.
\end{abstract}

1. Introduction. We are considering the mixed problem for the heat equation in one dimension:

$$
u_{t}=u_{x x}, \quad x \geqq 0, \quad 0 \leqq t \leqq T
$$

with the initial condition

$$
u(x, 0)=f(x), \quad x \geqq 0
$$

and boundary condition

$$
u_{x}(0, t)+\alpha u(0, t)=0, \quad 0 \leqq t \leqq T
$$

with $\alpha$ a constant. It is well known that this problem is well-posed in the maximum norm, i.e., there is a unique solution of (1a), (1b) satisfying

$$
\|u(\cdot, t)\|_{\infty}=\sup _{0 \leqq x}|u(x, t)| \leqq K^{\prime}(T)\|u(\cdot, 0)\|_{\infty}
$$

for $0 \leqq t \leqq T$.

We are concerned with the following finite-difference approximation to this problem: we introduce a mesh

$$
\begin{aligned}
x_{\nu} & =\nu h, & \nu & =1,2, \cdots \\
t_{n} & =n k, & n & =1,2, \cdots,[T / k]
\end{aligned}
$$

with $\lambda=k / h^{2}=$ constant, and solve

(2a) $v(\nu h,(n+1) k)=Q_{c} v(\nu h, n k), \quad \nu=1,2, \cdots, n=0,1, \cdots,[T / k]$

with initial condition

Received September 27, 1968, revised April 25, 1969.

AMS Subject Classifications. Primary 6568; Secondary 3578, 3515.

Key Words and Phrases. Stability, difference methods, mixed initial boundary-value problem, heat equation.

* This research was supported by a National Research Council of Canada post-doctoral fellowship at the University of Uppsala, Uppsala, Sweden and the National Physical Laboratory, Teddington, England, and by the Mathematics Research Center, United States Army, Madison, Wisconsin, under contract No: DA-31-124-ARO-D-462. 


$$
v(\nu h, 0)=f(\nu h), \quad \nu=1,2, \cdots
$$

where $Q_{c}=\sum_{-r}^{p} a_{j} E^{j}, E v(\nu h, t)=v(\nu h+h, t)$, and the $\left\{a_{j}\right\}$ are constants $\left(a_{p}\right.$, $\left.a_{-r} \neq 0\right)$. To specify $v$ completely, we must give $v(\mu h, n k)$ for $\mu=0,-1, \cdots$, $-r+1$. This we do as follows:

$$
v(\mu h, n k)=\sum_{j=1}^{l} b_{\mu j}(h) v(j h, n k), \quad \mu=0,-1, \cdots,-r+1 .
$$

Definition. The difference approximation (2a), (2b) is stable in the maximum norm if there exists a constant $K$ independent of $h$ such that for all $0 \leqq t=n k \leqq T$,

$$
\|v(\cdot, t)\|_{\infty}=\sup _{x=\nu h}|v(x, t)| \leqq K\|v(\cdot, 0)\|_{\infty} .
$$

We will give sufficient conditions for the difference approximation to be stable, using a technique due to Kreiss which he applies to hyperbolic systems of first order in [4]. We also analyze these conditions for a special case, and give corresponding sufficient conditions for stability in the case of two boundaries, $x=0$ and $x=1$ (strip problem).

2. Statement of the Main Theorem. We make the following assumptions about the difference approximation ( $2 \mathrm{a}),(2 \mathrm{~b})$ :

(a) Equations (2a), (2b) are consistent with (1a), (1b). That is, following Isaacson and Keller [2, p. 515], if we denote the differential equation by $L u=0$ with boundary condition $B u=0$, and the difference equation by $L_{h}(v)=0$ with boundary condition $B_{h}(v)=0$, then for all sufficiently smooth functions $w(x, t)$, we have $\left|\left(L-L_{h}\right) w(x, t)\right| \rightarrow 0$ as $h \rightarrow 0$ for $x \geqq 0,0 \leqq t \leqq T$, and $\left|\left(B-B_{h}\right) w(0, t)\right|$ $\rightarrow 0$ as $h \rightarrow 0$ for $0 \leqq t \leqq T$. In this case these conditions reduce to:

$$
\sum_{-r}^{p} a_{j}=1, \quad \sum j a_{j}=0, \quad \sum j^{2} a_{j}=2 \lambda
$$

and for the boundary conditions, using the homogeneity,

$$
\frac{1-\sum_{j=1}^{l} b_{\mu j}(h)}{\mu-\sum_{j=1}^{l} j b_{\mu j}(h)}=\alpha h+O\left(h^{2}\right) \text {. }
$$

We assume $c_{\mu}{ }^{\prime}(0)=\mu-\sum_{1}^{l} j b_{\mu j}(0) \neq 0$ for all $\mu$, although this is only for convenience. Following the technique used in [2, pp. 515-521], it is easy to show that the solution of (2a), (2b) converges to that of (1a), (1b) as $h \rightarrow 0$ if consistency and stability hold.

(b) The symbol (Fourier transform) of $Q_{c}, \hat{Q}_{c}(\xi)=\sum_{-r}^{p} a_{j} e^{i j \xi}$ satisfies $\left|\hat{Q}_{c}(\xi)\right| \leqq$ $1-d \xi^{2}$ for $|\xi| \leqq \pi$ for some $d>0$; i.e., $Q_{c}$ is a parabolic difference operator in the sense of Widlund [6]. This guarantees that the Cauchy problem is stable in the maximum norm. Because of (a), (b) is equivalent to assuming $\left|\hat{Q}_{c}(\xi)\right|<1$ for $\xi \neq 0$.

(c) The coefficients $\left\{b_{\mu j}(h)\right\}$ are $C^{2}(h)$ for $0 \leqq h \leqq h_{0}$. that

Let $Q(h)$ denote the operator (2a) complete with boundary condition (2b), so

$$
v(\nu h, n k)=(Q(h))^{n} f(\nu h)
$$


Then we can use the Dunford integral representation for $Q^{n}$ (see for example, Dunford and Schwartz [1, p. 568]):

$$
v(\nu h, n k)=\frac{-1}{2 \pi i}\left[\int_{\Gamma} z^{n}(Q-z I)^{-1} d z\right] f(\nu h) .
$$

Here $\Gamma$ is any path enclosing the spectrum of $Q(h)$. Note that we are considering $Q(h)$ as an operator on $l_{\infty}$ for $h>0$. To use (6), we must first examine the spectrum of $Q(h)$ in the neighborhood of the unit circle.

Lemma 1. Suppose $z$ belongs to $\operatorname{sp}(Q(h))$ and $|z| \geqq 1, z \neq 1$. Then $z$ is an eigenvalue of $Q(h)$, and for the matrix $B(z, h)$ given below, $\operatorname{det} B(z, h)=0$.

Proof. First consider such an eigenvalue $z$ of $Q(h)$ with eigenvector $g \in l_{\infty}$. Then we must have

$$
\begin{array}{ll}
\left(\sum_{-r}^{p} a_{j} E^{j}-z\right) g_{\nu}=0, & \nu=1,2, \cdots \\
g_{\mu}-\sum b_{\mu j}(h) g_{j}=0, & \mu=0,-1, \cdots,-r+1 .
\end{array}
$$

Thus $g$ has the form

$$
g_{\nu}=\sum_{\left|\tau_{j}\right| \leqq 1} p_{j}(\nu)\left(\tau_{j}(z)\right)^{\nu}
$$

where $\left\{\tau_{j}\right\}$ are the roots of $f(\tau)=\sum_{-r}^{p} a_{j} \tau^{j}=z$ and the $p_{j}(\nu)$ are polynomials in $\nu$ of degree one less than the multiplicity of $\tau_{j}$. Now, following Kreiss [4], if $\tau_{j}(z)=$ $e^{i \xi}, \xi \neq 0$, then

$$
|z|=\left|\sum_{-r}^{p} a_{j} e^{i j \xi}\right| \leqq 1-d \xi^{2}<1,
$$

and $\tau_{j}(z)=1$ means $z=1$, so there are no roots of $f(\tau)=z$ of modulus one for $|z| \geqq 1, z \neq 1$. For $|z|$ very large, Rouché's theorem shows that there are $r$ roots $\tau_{j}(z)$ with $\left|\tau_{j}\right|<1$, so, as the roots are continuous functions of $z$, there are exactly $r$ such roots for all $|z| \geqq 1, z \neq 1$. We call these $\tau_{1}, \cdots, \tau_{r}$.

Thus the eigenvector $g$ has $r$ independent parameters $\left\{\sigma_{j}\right\}_{1}{ }^{r}$ and it must satisfy $r$ homogeneous boundary conditions, giving the equations

$$
B(z, h) \text { d }=0 \text {. }
$$

Thus $z$ is an eigenvalue iff $\operatorname{det} B(z, h)=0$. For $z$ such that the $\left\{\tau_{j}(z)\right\}$ are distinct, we have $p_{j}(\nu) \equiv \sigma_{j}$ and

$$
B_{i j}(z, h)=\tau_{j}^{-i+1}-\sum_{k=1}^{l} b_{-i+1, k} \tau_{j}^{k} .
$$

Moreover, for $z$ with a multiple root $\tau_{j}(z)$, we must have $f^{\prime}\left(\tau_{j}\right)=0$, which is independent of $z$, so there are at most $r+p-1$ points $\left\{z_{i}{ }^{\prime}\right\}$ having multiple roots.

Now we claim that if $\operatorname{det} B(z, h) \neq 0$, then $(Q-z I)^{-1}$ exists on $l_{\infty}$, i.e., $z \notin$ sp $(Q(h))$. To see this, consider

$$
u(z)=(Q-z I)^{-1} f=\bar{u}(z)+w(z)
$$

where $\bar{u}(z)=\left(Q_{c}-z I\right)^{-1} f(f$ extended to $(-\infty, \infty)$ with same norm), and $w(z)$ is 
the correction required to fulfill the boundary conditions. From (b), we know $\bar{u}(z)$ exists for $|z| \geqq 1, z \neq 1$. Then $w(z)$ must satisfy

$$
\begin{aligned}
& \left(\sum_{-r}^{p} a_{j} E^{j}-z\right) w_{\nu}=0, \quad \nu=1,2, \cdots \\
& w_{\mu}-\sum_{j=1}^{l} b_{\mu j}(h) w_{j}=\tilde{f}_{\mu}=\sum_{1}^{l} b_{\mu j}(h) \bar{u}_{j}-\bar{u}_{\mu}, \quad \mu=0,-1, \cdots,-r+1 .
\end{aligned}
$$

Thus $w_{\nu}=\sum_{j=1}^{r} p_{j}{ }^{\prime}(\nu)\left(\tau_{j}(z)\right)^{\nu}$, with the $\left\{p_{j}{ }^{\prime}(\nu)\right\}$ having $r$ independent parameters $\left\{\sigma_{j}{ }^{\prime}\right\}$ with $B(z, h) \mathbf{d}^{\prime}=\tilde{f}$. Thus $w_{\nu}(z)$ exists (for all $f$ ) iff $\operatorname{det} B(z, h) \neq 0$ and in that case we have

$$
\left|w_{\nu}(z)\right| \leqq K_{1}\left\|B^{-1}(z, h)\right\|_{\infty} \nu^{r}(\rho(z))^{\nu}\left\|\left(Q_{c}-z I\right)^{-1}\right\|_{\infty}\|f\|_{\infty}
$$

where $\left|\tau_{j}(z)\right| \leqq \rho(z)<1$, giving

$$
\left\|(Q-z I)^{-1}\right\|_{\infty} \leqq\left\|\left(Q_{c}-z I\right)^{-1}\right\|_{\infty}\left(K_{1}+K_{2}\left\|B^{-1}(z, h)\right\|_{\infty}\right) .
$$

For the stability theorem, we also require the principal part $Q(0)$ of $Q(h)$, which is defined as the operator on $l_{\infty}$ obtained by taking $b_{\mu j}(0)$ for $b_{\mu j}$ in the operator. Then clearly a necessary condition for stability is that $Q(0)$ have no eigenvalues $z$ with $|z|>1$. Such a $z$ would be an isolated point in the spectrum of $Q(0)$ with finite multiplicity and so for small $h$ there would be an eigenvalue $z(h)$ of $Q(h)$ with $z(h) \rightarrow z$ as $h \rightarrow 0$. Thus from (5), using the eigenfunction of $z(h)$ as initial data, we could obtain an unbounded sequence $\|v(\cdot, T)\|_{\infty}$ as $h \rightarrow 0$.

We also assume that the roots $\tau_{j}(1)$ different from 1 are distinct; this is only for convenience in the proof-changes need only be made in the matrix $B(z, h)$ as we shall see in the proof.

Theorem 1. The difference approximation (2a), (2b) is stable in the maximum norm if the following conditions are satisfied:

(i) assumptions (a), (b), (c) hold;

(ii) the principal part $Q(0)$ has no eigenvalues $z$ with $|z| \geqq 1, z \neq 1$;

(iii) the matrix $B$ satisfies $\left\|(B(z, 0))^{-1}\right\|_{\infty} \leqq K_{0} /(|z-1|)^{1 / 2}$ for $|z-1| \leqq \delta_{0}$, $|z| \geqq 1$, for some $\delta_{0}>0$.

To motivate condition (iii), notice that the consistency conditions (3) imply that there is a double root of $f(\tau)=z$ at $\tau=1$ for $z=1$, and that these roots look like

$$
1 \pm\left(\frac{z-1}{\lambda}\right)^{1 / 2}+O(z-1)
$$

for $z$ close to 1 . So if we define $y(z)=((z-1) / \lambda)^{1 / 2}$ by a cut along the negative real axis from 1 to $-\infty$ (i.e., if $z=1+r e^{i \theta},(z-1)^{1 / 2}=r^{1 / 2} e^{i \theta / 2},-\pi<\theta \leqq 3 \pi$ ), we see that one root $\tau_{1}(z)$ is less than one in modulus and the other, $\tau_{r+1}(z)$ is greater than one in modulus. Then, using the consistency conditions (4),

$$
B_{\mu 1}(z, h)=\tau_{1}{ }^{\mu}-\sum_{1}^{l} b_{\mu j}(h) \tau_{1}{ }^{j}={c_{\mu}}{ }^{(}(\alpha h-y)+O\left(y^{2}\right)
$$

where again $c_{\mu}{ }^{\prime}(h)=\mu-\sum_{1}^{l} j b_{\mu j}(h)$. Thus for $z \rightarrow 1, B_{\mu 1}(z, 0) \rightarrow 0$ like $(z-1)^{1 / 2}$ 
and the simplest way for (iii) to hold is for the rest of $B$ to be nonsingular as $z \rightarrow 1$, i.e.

$$
\left.\operatorname{det}\left(\begin{array}{c}
c_{0}^{\prime}(0) \\
\vdots \\
c_{-r+1}^{\prime}(0)
\end{array}\right) b_{\mu i}=\left(\tau_{i}(1)\right)^{\mu}-\sum_{1}^{l} b_{\mu j}(0) \tau_{i}{ }^{j}\right) \neq 0 .
$$

Moreover, this can be related to the "generalized eigenvector" condition used by Kreiss in [4]. At $z=1$, we have a double root $\tau=1$, so there are $r+1$ parameters in the expression $B(1,0) \boldsymbol{\sigma}=0$ in Lemma 1 for an eigenvector of $Q-I$ with $r$ conditions to be met and the first column of $B$ zero. Now, however, the new root $\tau_{r+1}^{v}$ is $\nu \cdot(1)^{\nu}$, so the eigenvector is not necessarily in $l_{\infty}$, and the $(r+1)$ th column of $B(1,0)$ is precisely as given above: $\left(c_{0}^{\prime}(0), \cdots, c_{-r+1}^{\prime}(0)\right)^{T}$. So the determinant condition given above, which is sufficient for stability, is equivalent to $Q(0)$ not having any generalized eigenvectors at $z=1$ (generalized in the sense of polynomial growth) except the necessary one, $g_{\nu}=$ constant, corresponding to the solution $\boldsymbol{\delta}=(1,0, \cdots, 0)$ of $B \boldsymbol{d}=0$.

3. Proof of Theorem 1. The solution to (2a), (2b) can be written as

$$
v(\nu h, n k)=\sum_{\nu_{0}=1}^{\infty} \bar{v}\left(\nu h, n k ; \nu_{0} h\right) f\left(\nu_{0} h\right),
$$

where $\bar{v}\left(\nu h, n k ; \nu_{0} h\right)$ is the fundamental solution with initial condition

$$
\begin{aligned}
\bar{v}\left(\nu h, 0 ; \nu_{0} h\right)=\delta\left(\nu, \nu_{0}\right) & =0, & & \nu \neq \nu_{0}, \\
& =1, & & \nu=\nu_{0} .
\end{aligned}
$$

Thus

$$
\|v(\cdot, n k)\|_{\infty} \leqq\|f\|_{\infty} \cdot \sup _{\nu} \sum_{\nu_{0}=1}^{\infty}\left|\bar{v}\left(\nu h, n k ; \nu_{0} h\right)\right|,
$$

and we will show that this last term is uniformly bounded independent of $h$ and $\nu$ for all $0 \leqq t=n k \leqq T$. Of course, we only need to show this for $h$ small enough, since for $h>h_{0}$,

$$
\|v(\cdot, n k)\|_{\infty} \leqq\left\|Q^{n} f\right\|_{\infty} \leqq\|Q\|^{T / \lambda h_{0}^{2}}\|f\|_{\infty} .
$$

We will bound $\bar{v}\left(\nu h, n k ; \nu_{0} h\right)$ using (6). For this, we need an explicit bound for $u_{\nu}\left(z, \nu_{0}\right)=(Q-z I)^{-1} \delta\left(\nu, \nu_{0}\right)$ for $|z| \geqq 1, z \neq 1$. As in the proof of Lemma 1, we write this as

$$
u_{\nu}\left(z, \nu_{0}\right)=\bar{u}_{\nu}\left(z, \nu_{0}\right)+w_{\nu}\left(z, \nu_{0}\right)
$$

where $\bar{u}_{\nu}$ is the Cauchy solution and $w_{\nu}$ is the correction required to fulfill the boundary conditions.

First consider $\bar{u}_{\nu}\left(z, \nu_{0}\right)$. This satisfies

$$
\left(\sum_{-r}^{p} a_{j} E^{j}-z I\right) \bar{u}_{\nu}=\delta\left(\nu, \nu_{0}\right), \quad \nu-\nu_{0}=0, \pm 1, \pm 2, \cdots .
$$


From the proof of Lemma 1, we see that, apart from the points $\left\{z_{i}{ }^{\prime}\right\}, \bar{u}_{\nu}$ can be expressed as

$$
\begin{aligned}
\bar{u}_{\nu} & =\sum_{1}^{r} c_{j}\left(\tau_{j}(z)\right)^{\nu-\nu_{0}}, & \nu-\nu_{0}=1,2, \cdots \\
& =\sum_{r+1}^{r+p} c_{j}\left(\tau_{j}(z)\right)^{\nu-\nu_{0}}, & \nu-\nu_{0}=-1,-2, \cdots,
\end{aligned}
$$

where $\left\{\tau_{j}(z)\right\}$ are the roots of $f(\tau)=\sum_{-r}^{p} a_{j} \cdot \tau^{j}=z$ with $\left|\tau_{j}\right|<1$ for $j \leqq r$ and $\left|\tau_{j}\right|>1$ for $j>r$. Solving for the unknowns gives

$$
\begin{aligned}
\bar{u}_{\nu} & =\frac{1}{a_{p}} \sum_{k=1}^{r} \frac{\tau_{k}^{\nu-\nu_{0}+r-1}}{\prod_{j \neq k}\left(\tau_{k}-\tau_{j}\right)}, \quad \nu-\nu_{0}=0,1,2, \cdots \\
& =-\frac{1}{a_{p}} \sum_{k=r+1}^{r+p} \frac{\tau_{k}^{\nu-\nu_{0}+r-1}}{\prod_{j \neq k}\left(\tau_{k}-\tau_{j}\right)}, \quad \nu-\nu_{0}=0,-1,-2, \cdots .
\end{aligned}
$$

Note that as $z \rightarrow z_{i}{ }^{\prime} \neq 1\left(\left|z_{i}{ }^{\prime}\right| \geqq 1\right)$, where (say) $\tau_{i}=\tau_{k}(i<k \leqq r$ or $r<i$ $<k$ ), even though $c_{i} \rightarrow-c_{k} \rightarrow \infty$, we have

$$
\bar{u}_{\nu}\left(z, \nu_{0}\right) \rightarrow \frac{\left(\nu-\nu_{0}+\text { constant }\right) \tau_{i}{ }^{\nu-\nu_{0}}}{\prod_{j \neq i, k}\left(\tau_{i}-\tau_{j}\right)}+(\cdots)=\bar{u}_{\nu}\left(z_{i}{ }^{\prime}, \nu_{0}\right) .
$$

First consider $\bar{u}_{\nu}\left(z, \nu_{0}\right)$ for $z \in S_{\delta}=\{z:|z| \geqq 1,|z-1| \geqq \delta,|z| \leqq R\}$. Here we have for all roots $\tau_{j}(z),\left|\tau_{j}-1\right| \geqq$ constant $\cdot \sqrt{ } \delta$ (from consistency) and ||$\tau_{j}|-1| \geqq$ constant $\cdot\left(\arg \left(\tau_{j}\right)\right)^{2}$ (from parabolicity-see Widlund [6, Lemma 3.1]). Thus $\left|\tau_{j}\right| \leqq \rho(\delta)<1$ for $j<r$ and $\left|\tau_{j}\right|^{-1} \leqq \rho(\delta)<1$ for $j>r$. Finally this gives for $z \in S_{\delta}$,

$$
\left|\bar{u}_{\nu}\left(z, \nu_{0}\right)\right| \leqq\left|p\left(\nu-\nu_{0}\right)\right| \rho^{\left|\nu-\nu_{0}\right|} \leqq K_{1}(\delta) \rho_{1}^{\left|\nu-\nu_{0}\right|},
$$

where $p(x)$ is a polynomial, $\rho<\rho_{1}<1$, and $K_{1}$ is independent of $h$ and $\nu$.

Now consider $\bar{u}_{\nu}\left(z, \nu_{0}\right)$ for $z \in \bar{S}_{\delta}=\{z:|z| \geqq 1,|z-1| \leqq \delta\}$. As we showed earlier, we have $\tau_{1}(z) \rightarrow 1^{-}, \tau_{r+1}(z) \rightarrow 1^{+}$, and thus, since the other $\tau_{j}(z)$ are bounded away from one, we have for $|z-1| \leqq \delta_{1}$,

$$
\bar{u}_{\nu}\left(z, \nu_{0}\right)=\frac{g_{1}(z)(1-y)^{\left|\nu-\nu_{0}\right|}}{y}+M_{1}(z) \rho_{2}^{\left|\nu-\nu_{0}\right|}
$$

where $g_{1}(z)$ is analytic where the $\tau_{i}(z)$ are, $M_{1}(z)$ is bounded, and $\rho_{2}<1$.

Let us now examine the correction term $w_{\nu}\left(z, \nu_{0}\right)$ for $|z| \geqq 1, z \neq 1$. From the proof of Lemma 1, we see that apart from the exceptional points $\left\{z_{i}{ }^{\prime}\right\}$,

$$
w_{\nu}\left(z, \nu_{0}\right)=\sum_{j=1}^{r} d_{j} \tau_{j}^{\nu} \equiv\left(\tau^{\nu}, \mathrm{d}\right)
$$

where $\mathbf{d}$ satisfies $B(z, h) \mathbf{d}=\mathbf{t}\left(z, \nu_{0}\right)$, and

$$
\begin{aligned}
B_{\mu i} & =\tau_{i}{ }^{\mu}-\sum_{1}^{l} b_{\mu j}(h) \tau_{i}{ }^{j}, \quad i=1, \cdots, r \\
t_{\mu} & =\sum_{1}^{l} b_{\mu j}(h) \bar{u}_{j}\left(z, \nu_{0}\right)-\bar{u}_{\mu}\left(z, \nu_{0}\right)
\end{aligned}
$$


For an exceptional point $z_{i}{ }^{\prime}$ where (say) $\tau_{j}=\tau_{k}$, the same representation holds with $\tau_{k}{ }^{\nu}$ replaced by the independent solution $\nu \tau_{j}{ }^{\nu}$ in $B$ and $\tau^{\nu}$. We can also express $w_{\nu}$ as follows: let $\mathbf{s}^{\nu}$ be the solution of $B^{T} \mathbf{s}^{\nu}=\tau^{\nu}$. Then $w_{\nu}\left(z, \nu_{0}\right)=\left(\mathbf{s}^{\nu}, \mathbf{t}\right)$.

Again first consider $z \in S_{\delta}$. We know $B(z, 0)$ is nonsingular for all $|z| \geqq 1$, $z \neq 1$, continuous in $h$ for $h \leqq h_{0}$, and continuous in $z$ except at the $\left\{z_{i}{ }^{\prime}\right\}$. So if we take out small circles of radius $\epsilon$ around each $z_{i}{ }^{\prime} \in S_{\delta}$ and call the remainder $S_{\delta}{ }^{\prime}$, we have $\left\|B^{-1}(z, h)\right\|_{\infty} \leqq K_{2}(\delta, \epsilon)$ for $h \leqq h_{1}(\delta)$ and $z \in S_{\delta}{ }^{\prime}$. Moreover, near $z_{i}{ }^{\prime}$ where $\tau_{j}=\tau_{k}$, if we form $B^{\prime}(z, h)$ from $B(z, h)$ by subtracting column $j$ from column $k$ and dividing column $k$ by $\left(\tau_{k}-\tau_{j}\right)$ and likewise for $\tau^{\nu}$, we have $B^{\prime}(z, h) \rightarrow B\left(z_{i}{ }^{\prime}, h\right)$ as $z \rightarrow z_{i}{ }^{\prime}$ and thus $\mathbf{s}^{\nu}(z) \rightarrow \mathbf{s}^{\nu}\left(z_{i}{ }^{\prime}\right)$. Hence, for $h \leqq h_{2}(\delta)$ and $\epsilon$ small enough, we have $\left\|\left(B^{\prime}(z, h)\right)^{-1}\right\|_{\infty} \leqq K_{3}(\delta, \epsilon)$ in $S_{\delta}-S_{\delta}^{\prime}$. So in all of $S_{\delta}$, using (8) and the fact that $\mathrm{t}$ is bounded in $S_{\delta}$, we have

$$
\left|w_{\nu}\left(z, \nu_{0}\right)\right| \leqq K_{3}(\delta)\left(\rho_{1}(\delta)\right)^{\left|\nu-\nu_{0}\right|}
$$

for all $z \in S_{\delta}$, where $K_{3}(\delta)$ is independent of $h$ and $\nu$.

Now examine $w_{\nu}\left(z, \nu_{0}\right)$ for $z \in \bar{S}_{\delta}$. Here we need that the other roots $\tau_{j}(1)$, $j \neq 1, r+1$ are distinct; but if they are not, we merely work with $B^{\prime}(z, h)$ as before, and require that condition (iii) hold for $B^{\prime}(z, 0)$. We claim that $B(z, h)$ is nonsingular in the region $S_{h}=\left\{z: c h^{2} \leqq|z-1| \leqq \delta,|z| \geqq 1\right\}$ for $0<h \leqq h_{0}$ and some constant $c$, if condition (iii) holds. To see this, observe that we can write $B(z, h)=$ $B(z, 0)+H(h)$, where $\|H(h)\|_{\infty} \leqq k_{1} h$. Then in $S_{h}$

$$
\left\|(B(z, 0))^{-1} H\right\| \leqq K_{0} K_{1} / c<1 \text { for } c>K_{0} K_{1} .
$$

Thus $(B(z, h))^{-1}=\left(I+(B(z, 0))^{-1} H(h)\right)^{-1}(B(z, 0))^{-1}$ exists and in fact in $S_{h}$,

$$
\left\|(B(z, h))^{-1}\right\| \leqq \frac{K_{0}}{\left(1-K_{0} K_{1} / c\right)(|z-1|)^{1 / 2}} .
$$

Actually, we can prove the above with a little less: we can have

$$
\left|(B(z, 0))_{1 i}^{-1}\right| \leqq K_{0} /|z-1|, \quad i=1, \cdots, r,
$$

and

$$
\left|(B(z, 0))_{\mu i}^{-1}\right| \leqq K_{0} /(|z-1|)^{1 / 2}
$$

for the other rows. Because of the consistency conditions (4), we have

$$
B_{\mu 1}(z, 0)=c_{\mu}{ }^{\prime}(0) \cdot y(z)+O(z-1), \quad H_{\mu 1}(h)=-h \alpha c_{\mu}{ }^{\prime}(0)+O(h y)
$$

so that

$$
\left(B^{-1} H\right)_{11}=O(h / y), \quad\left(B^{-1} H\right)_{1 i}=O\left(h / y^{2}\right), \quad\left(B^{-1} H\right)_{i 1}=O(h),
$$

and thus using a diagonal similarity transformation $D=\operatorname{diag}(y, 1, \cdots, 1)$, we have $\left\|D B^{-1} H D^{-1}\right\| \leqq K^{\prime} / c<1$ for $c$ large enough, implying the existence of $(B(z, h))^{-1}$.

Thus $w_{\nu}\left(z, \nu_{0}\right)$ exists in $S_{h}$ for all $\nu_{0}$. Now we can proceed to estimate it. From (9) and (4), we have 


$$
\begin{aligned}
t_{\mu}= & \frac{g_{1}(z)(1-y)^{\nu_{0}}}{y}(-h \alpha-y) c_{\mu}{ }^{\prime}(h)+K_{\mu}(z) y(1-y)^{\nu_{0}} \\
& +L_{\mu}\left(z, \nu_{0}\right)(1-y)^{\nu_{0}}+K_{\mu}{ }^{\prime}(z) \rho_{2}{ }^{\nu_{0}},
\end{aligned}
$$

where $K_{\mu}(z), K_{\mu}{ }^{\prime}(z)$ are bounded and $L_{\mu}\left(z, \nu_{0}\right)$ is bounded and identically zero for $\nu_{0} \geqq l$. Then from (6) we have

$$
\begin{aligned}
t_{\mu}= & \frac{-g_{1}(z)(1-y)^{\nu_{0}}}{y}\left(\frac{h \alpha+y}{h \alpha-y}\right) B_{\mu 1}(z, h)+K_{\mu}^{\prime \prime}(z) y(1-y)^{\nu_{0}} \\
& +L_{\mu}\left(z, \nu_{0}\right)(1-y)^{\nu_{0}}+K_{\mu}{ }^{\prime}(z) \rho_{2}^{\nu_{0}}
\end{aligned}
$$

Thus

$$
\begin{aligned}
w_{\nu}\left(z, \nu_{0}\right) & =\left(\tau^{\nu}, B^{-1} \mathrm{t}\right) \\
& =\frac{-g_{1}(z)(1-y)^{\nu+\nu_{0}}}{y}\left(\frac{h \alpha+y}{h \alpha-y}\right)+M_{2}\left(z, \nu_{0}\right),
\end{aligned}
$$

where $\sum_{\nu_{0=1}}^{\infty}\left|M_{2}\left(z, \nu_{0}\right)\right| \leqq K /|y(z)|$. Notice that here we use (iii) fully.

Now return to the fundamental solution $\bar{v}\left(\nu h, n k ; \nu_{0} h\right)$. From our analysis of $\bar{u}_{\nu}$ and $w_{\nu}$, we have

$$
\bar{v}\left(\nu h, n k ; \nu_{0} h\right)=\frac{-1}{2 \pi i} \int_{\Gamma_{1}+\Gamma_{2}} z^{n}\left(\bar{u}_{\nu}\left(z, \nu_{0}\right)+w_{\nu}\left(z, \nu_{0}\right)\right) d z
$$

where $\Gamma_{1}$ and $\Gamma_{2}$ are given by

$$
\begin{aligned}
\Gamma_{2}: z & =e^{i \theta},|z-1| \geqq \delta \\
\Gamma_{1}: z & =e^{i \theta}, \operatorname{ch}^{2} \leqq|z-1| \leqq \delta \\
z & =1+\operatorname{ch}^{2} e^{i \phi},-\pi / 2+O(\delta) \leqq \phi \leqq \pi / 2+O(\delta) .
\end{aligned}
$$

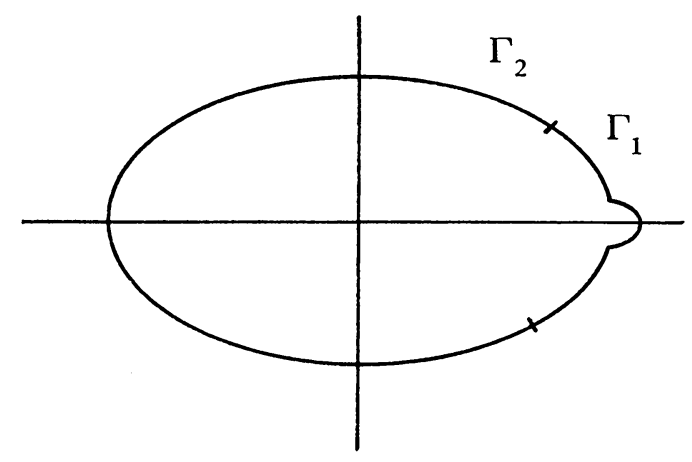

Using (8), (9), (10), (11), we see

$\sum_{\nu_{0}}\left|\bar{v}\left(\nu h, n k ; \nu_{0} h\right)\right|$

$$
\leqq K_{4}(\delta)+\frac{1}{2 \pi} \sum_{\nu 0}\left|\int_{\Gamma} \frac{z^{n} g_{1}(z)}{y}\left[(1-y)^{\left|\nu-\nu_{0}\right|}-\left(\frac{h \alpha+y}{h \alpha-y}\right)(1-y)^{\nu+\nu_{0}}\right] d z\right|
$$


where $K_{4}$ is independent of $h, n$, and $\nu$.

Now we will estimate this integral by contour integration. Recall that $g_{1}(z)$, defined by (9), is analytic where the roots $\tau_{i}(z)$ are analytic. Although (9) defines $g_{1}(z)$ only for $|z-1| \leqq \delta,|z| \geqq 1$, it is actually a well-defined analytic function in all of $|z-1| \leqq \delta$ except for the cut along the negative real axis, since the $\tau_{i}(z)$ are well-defined analytic functions there. Here we assume $\delta$ is so small that there are no exceptional points $\left\{z_{i}{ }^{\prime}\right\}$ inside $|z-1| \leqq \delta$ except $z=1$. Moreover, we assumed for convenience that the other roots $\tau_{i}(1)$ different from 1 were distinct; if not, we can again modify the argument by taking definite branches of the multiple root.

In particular, we consider the paths

$$
\begin{aligned}
& \Gamma_{2}: z=1+\delta e^{i \theta}, \theta_{1} \leqq|\theta| \leqq 2 \pi / 3 \text { with }\left|z\left(\theta_{1}\right)\right|=1\left(\theta_{1}=\pi / 2+O(\delta)\right) \\
& \Gamma_{4}: z=1-2 \delta(1-\cos \phi) e^{-i \phi},-\pi / 3 \leqq \phi \leqq \pi / 3 .
\end{aligned}
$$

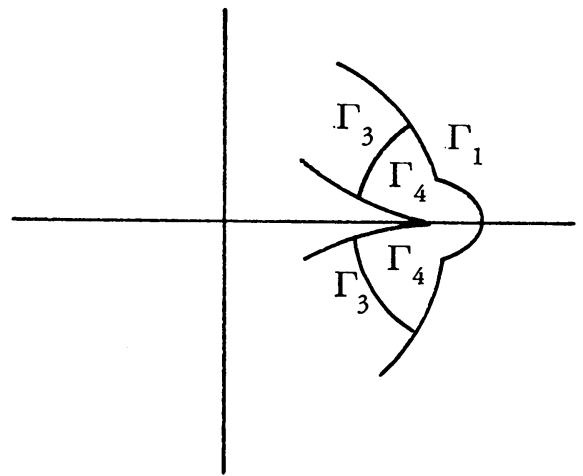

As a function of $y=((z-1) / \lambda)^{1 / 2}$, the integrand is a single-valued analytic function inside and on the closed contour $\Gamma_{1}+\Gamma_{3}+\Gamma_{4}$, with a simple pole at $y=h \alpha$ (which is inside the contour if $\alpha>0$ ). Thus

$$
\frac{1}{2 \pi} \sum_{\nu_{0}}\left|\int_{\Gamma_{1}+\Gamma_{3}+\Gamma_{4}}\right|=\sum_{\nu_{0}} \mid \text { residues }|\leqq 2| g_{1}\left(1+\lambda h^{2} \alpha^{2}\right) \mid\left(1+\lambda h^{2} \alpha^{2}\right)^{n} .
$$

Now we will bound the integrals on $\Gamma_{3}+\Gamma_{4}$. First note that since $\bar{v}\left(\nu h, n k ; v_{0} h\right)$ $=(Q(h))^{n} \delta\left(\nu, \nu_{0}\right)=0$ for $\left|\nu-\nu_{0}\right|>q n$ where $q=\max (r, p)$, we need only sum over these $\nu_{0}$. (This also means the analysis holds only for explicit schemes.) We have

$$
\sum_{\nu_{0}}\left|\int_{\Gamma_{3}+\Gamma_{4}}\right| \leqq \sum_{m=1}^{q n}\left|\int_{\Gamma_{3}+\Gamma_{4}} g_{2}(z) \frac{z^{n}(1-y)^{m}}{y} d z\right|,
$$

where $g_{2}(z)$ is analytic and bounded independent of $h, \nu$, and $n$. On $\Gamma_{3},|1-y| \leqq$ $1-(\delta / \lambda)^{1 / 2}$ which gives

$$
\sum_{m=1}^{q n}\left|\int_{\Gamma_{3}}\right| \leqq \lambda \pi \cdot \max _{\Gamma_{3}}\left|g_{2}(z)\right| .
$$

On $\Gamma_{4}$, the integral can be expressed as follows:

$$
2 i(\lambda \delta)^{1 / 2} \int_{-\pi / 3}^{\pi / 3} g_{2}(z)\left(1-2 \delta(1-\cos \phi) e^{-i \phi}\right)^{n}\left(1-\left(\frac{\delta}{\lambda}\right)^{1 / 2}\left(1-e^{-i \phi}\right)\right)^{m} e^{-i \phi} d \phi,
$$


since $y=((z-1) / \lambda)^{1 / 2}=(\delta / \lambda)^{1 / 2}\left(1-e^{-i \phi}\right)$ on this path. We can estimate this integral following John [3, p. 119]. Let $z(\phi)=1-2 \delta(1-\cos \phi) e^{-i \phi} \equiv e^{-f(\phi)}$, defined by the principal value of the logarithm. Then $f(\phi)$ is analytic for $|\phi| \leqq \pi / 3$ and for $\phi \rightarrow 0, f(\phi)=-\log z=\delta \phi^{2}+O\left(\phi^{3}\right)$. Then $\left|e^{-f(\phi)}\right| \leqq \exp \left[-\delta^{\prime} \phi^{2}\right]$ for $|\phi| \leqq \pi / 3$, since for $|\phi| \leqq \phi_{0}$ (say), $-\left(\log |z| / \phi^{2}\right) \geqq \delta / 2$ and for $\phi_{0} \leqq|\phi| \leqq \pi / 3$, $-(\log |z|) / \phi^{2} \geqq \delta^{\prime \prime}>0$, so we can take $\delta^{\prime}=\min \left(\delta^{\prime \prime}, \delta / 2\right)$.

Using this, we have for any $\epsilon>0$,

$$
\left|\int_{\epsilon \leqq|\phi|<\pi / 3}\right| \leqq 2 \cdot \max _{\Gamma_{4}}\left|g_{2}(z)\right| \cdot \int_{\epsilon}^{\infty} \exp \left[-\delta^{\prime} n \phi^{2}\right] d \phi \leqq K_{5}(\delta) \frac{\exp \left[-\delta^{\prime} n \epsilon^{2}\right]}{n \epsilon} .
$$

For $|\phi| \leqq \epsilon,(z(\phi))^{n}=\exp \left(-\delta n \phi^{2}+O\left(n \phi^{3}\right)\right)$, and

$$
(1-y(\phi))^{m}=\exp \left(-i m \delta_{1}{ }^{\prime} \phi-m \delta_{2}{ }^{\prime} \phi^{2}+O\left(m \phi^{3}\right)\right),
$$

where $\delta_{1}{ }^{\prime}=(\delta / \lambda)^{1 / 2}$ and $\delta_{2}{ }^{\prime}=\frac{1}{2} \delta_{1}{ }^{\prime}-\delta / \lambda$. Now take $\epsilon=n^{-1 / 3}$ and $m^{\prime}=\delta n+\delta_{2}{ }^{\prime} m$. Then, since $m \leqq q n$,

$$
\begin{aligned}
\left|\int_{\Gamma_{4}}\right| \leqq & \int_{-\infty}^{\infty} \exp \left(-i m \delta_{1}{ }^{\prime} \phi-m^{\prime} \phi^{2}\right) d \phi+K_{6}(\delta) \int_{0}^{\epsilon} \exp \left[-\delta_{3}{ }^{\prime} n \phi^{2}\right] O\left(n \phi^{3}\right) d \phi \\
& +K_{7}(\delta) \frac{\exp \left[-\delta_{4}{ }^{\prime} n \epsilon^{2}\right]}{n \epsilon} \\
= & \left(\frac{\pi}{m^{\prime}}\right)^{1 / 2} \exp \left(-\left(m \delta_{1}{ }^{\prime}\right)^{2} / 4 m^{\prime}\right)+O\left(\frac{1}{n}\right) \\
\leqq & \frac{K_{8}(\delta)}{(n c)^{1 / 2}} \exp \left(-m^{2} / n c\right)+O\left(\frac{1}{n}\right)
\end{aligned}
$$

and thus $\sum_{m=1}^{q n}\left|\int_{\Gamma_{4}}\right| \leqq K_{9}(\delta)$, independent of $h, n$, and $\nu$. Finally this, together with (12) and our previous estimates, gives

$$
\sum_{\nu_{0}}\left|\bar{v}\left(\nu h, n k ; \nu_{0} h\right)\right| \leqq K_{10}(\delta)\left(1+\lambda h^{2} \alpha^{2}\right)^{n} \leqq K_{11} e^{c^{\prime} T}
$$

which completes the proof of Theorem 1 . Also note that if $\alpha \leqq 0$, we can take $c^{\prime}=0$, i.e., the solution is stable for all time $T$, for $h$ small enough.

4. Stability for the 3-Point Scheme. For a general scheme with general boundary conditions, it may be difficult to check condition (ii) of Theorem 1. However, in special cases of practical significance, it is fairly easy. In particular, consider the well-known 3-point scheme: $a_{-1}=a_{1}=\lambda, a_{0}=1-2 \lambda, 0<\lambda<1 / 2$. We need a boundary condition of the form $v(0, t)=\sum_{1}^{l} b_{j}(h) v(j h, t)$. Let us consider $b_{j}(h)=$ $b_{j}(0) / g(h), g(h)=1+h g^{\prime}(0)+\cdots$, and demand that the coefficients be chosen so that the boundary condition agrees with that for the differential equation to as high an order of accuracy as possible. We have, if $u(x, t)$ is the solution to the differential equation,

$$
h g(h) B_{h} u(0, t)=u(0, t) g(h)-\sum_{k=0}^{\infty} \frac{c_{k} h^{k}}{k !} \frac{\partial^{k} u(0, t)}{\partial x^{k}},
$$

where $c_{k}=\sum_{1}^{l} j^{k} b_{j}(0)$. Consistency requires $c_{0}=1, c_{1}=-g^{\prime}(0) / \alpha$, and we can equate to zero the coefficients of $h^{i}$ by taking $g^{(i)}(0)=0, c_{i}=0$ for $2 \leqq i \leqq l$. 
This gives the solution

$$
b_{j}(h)=\frac{(-1)^{j-1}\left(\begin{array}{l}
l \\
j
\end{array}\right)}{j\left(\sum_{1}^{l}(1 / k)-h \alpha\right)} .
$$

Theorem 2. For the explicit 3-point scheme with these boundary conditions, the operator $Q(0)$ has no eigenvalues $z$ with $|z| \geqq 1, z \neq 1$, for $\lambda$ small enough.

Proof. The equation $\operatorname{det} B(z, 0)=0$ is in this case a polynomial in $\tau$ :

$$
B(z, 0)=\frac{p(\tau)}{\sum_{1}^{l} 1 / k}=1-\sum_{1}^{l} b_{j}(0) \tau^{j} .
$$

If there is an eigenvalue $z,|z| \geqq 1, z \neq 1$, we must have $z=1+\lambda(\tau+1 / \tau-2)$, for $\tau$ some root of $p(\tau)=0$ with $|\tau| \leqq 1$. First of all, we claim there are no roots of $p(\tau)=0$ for $|\tau-1| \leqq 1$ except $\tau=1$. To see this, notice that $p^{\prime}(\tau)=$ $\left((1-\tau)^{l}-1\right) / \tau$, which means $p^{\prime}(\tau)=-\sum_{0}^{l-1}(1-\tau)^{i}$ and thus

$$
p(\tau) /(1-\tau)=\sum_{1}^{l}(1-\tau)^{i-1} / i
$$

which has no roots in $|\tau-1| \leqq 1$ by the Eneström-Kakeya theorem (Marden [5, p. 136]).

Now let $\tau=x+i y$ be some root of $p(\tau)=0$ with $|\tau| \leqq 1$. Then

$$
\begin{aligned}
z(\tau) & =1+\lambda\left(x+\frac{x}{x^{2}+y^{2}}-2\right)+i \lambda\left(y-\frac{y}{x^{2}+y^{2}}\right) \\
& =1-\lambda q(\tau)+i \lambda r(\tau),
\end{aligned}
$$

and $q(\tau)=2-x-x /\left(x^{2}+y^{2}\right)>0$ since $|\tau-1|>1$. Then $|z(\tau)|<1$ if $\lambda<$ $2 q(\tau) /\left(q^{2}(\tau)+r^{2}(\tau)\right)$. So finally, for

$$
\lambda<\min _{\tau \text { roots }} \frac{2 q(\tau)}{q^{2}(\tau)+r^{2}(\tau)},
$$

all eigenvalues of $Q(0)$ are less than one in modulus, except for $z=1$. Q.E.D.

Similar results can be shown for special cases of higher-order approximations. For example, it is easy to show that there are no roots $\tau$ of $\operatorname{det} B(z, 0)=0$ with $|\tau|<1$ (and thus no eigenvalues $z$ with $|z| \geqq 1, z \neq 1$ ) for schemes with $r=2$ and the simple consistent boundary conditions $D_{+} u(0, t)+\alpha u(0, t)=0, D_{+}{ }^{2} u(-h, t)$ $=0$, where $D_{+} u(x, t)=(u(x+h, t)-u(x, t)) / h$.

5. The Strip Problem. The preceding analysis can be extended to give stability conditions for the mixed problem with two boundaries:

$$
\begin{aligned}
u_{t} & =u_{x x}, \quad 0 \leqq x \leqq 1, \quad 0 \leqq t \leqq T \\
u(x, 0) & =f(x), \quad 0 \leqq x \leqq 1, \\
u_{x}(0, t)+\alpha u(0, t) & =0, \quad u_{x}(1, t)+\beta u(1, t)=0, \quad 0 \leqq t \leqq T .
\end{aligned}
$$

The corresponding finite-difference approximation is (with $N h=1$ ): 


$$
\begin{aligned}
v(\nu h, n k+k) & =\sum_{-r}^{p} a_{j} v(\nu h+j h, n k), \quad \nu=1,2, \cdots, N-1 \\
v(\nu h, 0) & =f(\nu h) \\
v(\mu h, n k) & =\sum_{1}^{l} b_{\mu j}(h) v(j h, n k), \quad \mu=0,-1, \cdots,-r+1 \\
v(N h+\mu h, n k) & =\sum_{1}^{m} c_{\mu j}(h) v(N h-j h, n k), \quad \mu=0,1, \cdots, p-1 .
\end{aligned}
$$

Both problems can be thought of as combinations of right and left half-plane problems of the kind described in the first part of the paper. In fact we have

THEOREM 3. The finite-difference scheme (15) is stable in the maximum norm if the conditions of Theorem 1 hold for both the left and right half-plane problems.

Proof. As before, we can express the solution of (15) as $v(\nu h, n k)=(Q(h))^{n} f(\nu h)$, where $Q(h)$ now operates on $N$-vectors, and thus

$$
\|v(\cdot, n k)\|_{\infty} \leqq\|f\|_{\infty} \sup _{\nu} \sum_{\nu_{0}=1}^{N-1}\left|\bar{v}\left(\nu h, n k ; \nu_{0} h\right)\right|,
$$

and

$$
\bar{v}\left(\nu h, n k ; \nu_{0} h\right)=\frac{-1}{2 \pi i} \int_{\Gamma} z^{n}(Q(h)-z I)^{-1} \delta\left(\nu, \nu_{0}\right) d z
$$

Let us write

$$
(Q(h)-z I)^{-1} \delta\left(\nu, \nu_{0}\right)=u_{\nu}\left(z, \nu_{0}\right)=\bar{u}_{\nu}\left(z, \nu_{0}\right)+r_{\nu}\left(z, \nu_{0}\right)
$$

where again $\bar{u}_{\nu}$ is the Cauchy solution and $r_{\nu}\left(z, \nu_{0}\right)$ is the correction required for the boundary conditions. Now let $r_{\nu}=w_{\nu}+s_{\nu}$, where $w_{\nu}$ and $s_{\nu}$ are the right and left half-plane eigenfunctions, so that apart from exceptional points,

$$
w_{\nu}=\sum_{1}^{r} \sigma_{j} \tau_{j}^{\nu}, s_{\nu}=\sum_{r+1}^{r+p} \sigma_{j} \tau_{j}^{\nu-N} .
$$

Using the notation of Theorem 1 , solving for the $\left\{\sigma_{j}\right\}$ gives

$$
s_{\nu}+w_{\nu}=\left(\tau_{1}{ }^{\nu}, \tau_{2}{ }^{\nu-N}\right)\left(\begin{array}{ll}
B_{1} & E_{1} \\
E_{2} & B_{2}
\end{array}\right)^{-1}\left(\begin{array}{c}
\mathrm{t} \\
\mathrm{t}^{\prime}
\end{array}\right)
$$

where

$$
\begin{aligned}
&\left(B_{1}\right)_{\mu i}=\tau_{i}{ }^{\mu}-\sum_{1}^{l} b_{\mu j} \tau_{i}{ }^{j}, \quad \mu=0,-1, \cdots,-r+1, i=1, \cdots, r \\
&\left(E_{1}\right)_{\mu i}=\tau_{i}{ }^{-N}\left(\tau_{i}{ }^{\mu}-\sum_{1}^{l} b_{\mu j} \tau_{i}{ }^{j}\right), \mu=0,-1, \cdots,-r+1, \\
& i=r+1, \cdots, r \\
&\left(E_{2}\right)_{\mu i}=\tau_{i}{ }^{N}\left(\tau_{i}{ }^{\mu}-\sum_{1}^{m} c_{\mu j} \tau_{i}{ }^{-j}\right), \quad \mu=0,1, \cdots, p-1, i=1, \cdots, r \\
&\left(B_{2}\right)_{\mu i}=\tau_{i}{ }^{\mu}-\sum_{1}^{m} c_{\mu j} \tau_{i}{ }^{-j}, \quad \mu=0,1, \cdots, p-1, \quad i=r+1, \cdots, r+p
\end{aligned}
$$




$$
\begin{aligned}
t_{\mu} & =\sum_{1}^{l} b_{\mu j} \bar{u}_{j}-\bar{u}_{\mu}, \quad \mu=0,-1, \cdots,-r+1 \\
t_{\mu}{ }^{\prime} & =\sum_{1}^{m} c_{\mu j} \bar{u}_{N-j}-\bar{u}_{N+\mu}, \quad \mu=0,1, \cdots, p-1 .
\end{aligned}
$$

Note that if $E_{1}=E_{2}=0$, we obtain the solutions for the separate right and left half-plane problems.

Clearly, for $|z-1| \geqq \delta$, the matrix inverse exists for $h$ small enough since $E_{1}$ and $E_{2}$ are very small, and indeed we have an estimate like (10) for $\left(s_{\nu}+w_{\nu}\right)$. For $|z-1| \leqq \delta$, the situation is more delicate as the first columns of all four matrices approach zero similarly. We can write

$$
\left(\begin{array}{ll}
B_{1} & E_{1} \\
E_{2} & B_{2}
\end{array}\right)^{-1}=\left(\begin{array}{cc}
\left(I-H_{1}\right)^{-1} B_{1}{ }^{-1} & -B_{1}^{-1} E_{1}\left(I-H_{2}\right)^{-1} B_{2}^{-1} \\
-B_{2}{ }^{-1} E_{2}\left(I-H_{1}\right)^{-1} B_{1}^{-1} & \left(I-H_{2}\right)^{-1} B_{2}{ }^{-1}
\end{array}\right)
$$

where $H_{1}=B_{1}^{-1} E_{1} B_{2}^{-1} E_{2}$ and $H_{2}=B_{2}^{-1} E_{2} B_{1}^{-1} E_{1}$. Using the consistency conditions

$$
\begin{aligned}
& \frac{1-\sum_{1}^{l} b_{\mu j}(h)}{\mu-\sum_{1}^{l} j b_{\mu j}(h)}=h \alpha+O\left(h^{2}\right), \quad \mu=0,-1, \cdots,-r+1, \\
& \frac{1-\sum_{1}^{m} c_{\mu j}(h)}{\mu+\sum_{1}^{m} j c_{\mu j}(h)}=h \beta+O\left(h^{2}\right), \quad \mu=0,1, \cdots, p-1,
\end{aligned}
$$

we can write, using $y=((z-1) / \lambda)^{1 / 2}$,

$$
B_{1}^{-1} E_{1}=g_{3}(z) e_{1} e_{1}{ }^{T}+O(y), \quad B_{2}{ }^{-1} E_{2}=g_{4}(z) e_{1} e_{1}{ }^{T}+O(y),
$$

where

$$
g_{3}(z)=(1-y)^{N}\left(\frac{h \alpha+y}{h \alpha-y}\right), \quad g_{4}(z)=(1-y)^{N}\left(\frac{h \beta-y}{h \beta+y}\right) .
$$

Thus on the contour $\Gamma_{1}$ used in the proof of Theorem 1, we had $|y| \geqq c^{\prime} h$ and thus on this path, $\left|H_{i}(z, h)\right| \leqq K e^{-2 c^{\prime}}<1$ for $c^{\prime}$ large enough $(i=1,2)$. So the matrix inverse exists on such a contour, and in fact we have

$$
w_{\nu}+s_{\nu}=\bar{w}_{\nu}\left(1+g_{5}(z)\right)+\bar{s}_{\nu}\left(1+g_{6}(z)\right)+M_{3}\left(z, \nu_{0}\right),
$$

where $M_{3}\left(z, \nu_{0}\right)$ is again harmless, $\bar{w}_{\nu}$ and $\bar{s}_{\nu}$ are the corresponding solutions for the separate right and left half-plane problems, and $g_{5}(z), g_{6}(z)$ are meromorphic in $y$ with isolated singularities at $y=h \alpha$ and $y==h \beta$. Thus we can use the same technique of contour integration to bound $\sum_{\nu_{0}}\left|\bar{v}\left(\nu h, n k ; \nu_{0} h\right)\right|$, and indeed the same contour. We merely obtain a different residue at the isolated singularities, giving

$$
\sum_{\nu_{0}}\left|\bar{v}\left(\nu h, n k ; \nu_{0} h\right)\right| \leqq K \cdot\left[\left(1+\lambda h^{2} \alpha^{2}\right)\left(1+\lambda h^{2} \beta^{2}\right)\right]^{n} \leqq K^{\prime} e^{c^{\prime \prime} T} .
$$

Note also that if $\alpha \leqq 0$ and $\beta \geqq 0$, the singularities are not inside the contour and thus the bound holds for all time $T$. As the referee mentioned, in this case the underlying differential equation satisfies a maximum principle.

Acknowledgement. I am indebted to Heinz-Otto Kreiss for suggesting this problem to me, and to both him and Olof Widlund for their assistance and encouragement. 
Applied Mathematics Department California Institute of Technology Pasadena, California 91109

1. N. Dunford \& J. T. Schwartz, Linear Operators. Part I: General Theory, Pure and Appl. Math., vol. 7, Interscience, New York, 1958. MR 22 \#8302.

2. E. IsaAcson \& H. B. Keller, Analysis of Numerical Methods, Wiley, New York, 1966. MR 34 \#924.

3. F. JoHn, Lectures on Advanced Numerical Analysis, Gordon \& Breach, New York, 1967. MR $36 \# 4773$.

4. H.-O. KREISs, "Stability theory for difference approximations of mixed initial boundary value problems. I," Math. Comp., v. 22, 1968, pp. 703-714.

5. M. MARDEN, Geometry of Polynomials, 2nd ed., Amer. Math. Soc. Math. Surveys, no. 3, Amer. Math. Soc., Providence, R. I., 1966. MR 37 \#1562.

6. O. B. WIDLUND, "Stability of parabolic difference schemes in the maximum norm," Numer. Math., v. 8, 1966, pp. 186-202. MR 33 \#5149. 